\title{
Wave Equations with Time-dependent Spatial Operators of Higher Order
}

\author{
Peter Lesky Jr \\ Mathematisches Institut A, Universität Stuttgart, Pfaffenwaldring 57, W-7000, Stuttgart 80, \\ Germany
}

Communicated by $\mathbf{R}$. Leis

We study the initial-boundary value problem for $\partial_{t}^{2} u(t, x)+A(t) u(t, x)+B(t) \partial_{2} u(t, x)=f(t, x)$ on $[0, T]$ $\times \Omega\left(\Omega \subset \mathbb{R}^{n}\right)$ with a homogeneous Dirichlet boundary condition; here $A(t)$ denotes a family of uniformly strongly elliptic operators of order $2 m, B(t)$ denotes a family of spatial differential operators of order less than or equal to $m$, and $u$ is a scalar function. We prove the existence of a unique strong solution $u$. Furthermore, an energy estimate for $u$ is given.

\section{Introduction}

Let $\Omega \subset \mathbb{R}^{n}$ and $m \in \mathbb{N}$ be given. We consider the problem

$$
\left.\begin{array}{ll}
\partial_{t}^{2} u(t, x)+A(t) u(t, x)+B(t) \partial_{t} u(t, x)=f(t, x) & \text { for } t \in[0, T], x \in \Omega, \\
u(t, .) \in \mathscr{H}^{m}(\Omega) & \text { for } t \in[0, T], \\
u(0, x)=u^{0}(x), \quad \partial_{\imath} u(0, x)=u^{1}(x) & \text { for } x \in \Omega .
\end{array}\right\}
$$

Here $\stackrel{\circ}{H}^{m}(\Omega)$ denotes the closure of $C_{0}^{\infty}(\Omega)$ in the $m$ th Sobolev space $H^{m}(\Omega)$, and $A(t), B(t)$ denote families of spatial differential operators of order $2 m$ and less than or equal to $m$, respectively. Problems of this kind appear in the study of fully non-linear wave equations (compare [9]). We make the following assumptions.

Assumption 1.1. (1) The operators $A$ and $B$ are given by

$$
\begin{array}{ll}
A(t) \varphi:=\sum_{|\alpha| \leqslant 2 m}\left[a_{\alpha}(t, .)+\tilde{a}_{\alpha}(t, .)\right] \partial_{x}^{\alpha} \varphi & \text { for } \varphi \in \stackrel{H}{H}^{m}(\Omega) \cap H^{2 m}(\Omega), \\
B(t) \varphi:=\sum_{|\beta| \leqslant m}\left[b_{\beta}(t, .)+\tilde{b}_{\beta}(t, .)\right] \partial_{x}^{\beta} \varphi \quad \text { for } \varphi \in H^{m}(\Omega),
\end{array}
$$

where $a_{\alpha}, b_{\beta} \in C_{b}^{(k-1) m}([0, T] \times \bar{\Omega})$ and

$$
\tilde{a}_{\alpha}, \tilde{b}_{\beta} \in \bigcap_{j=1}^{k-1} C^{j}\left([0, T], H^{(k-1-j) m}(\Omega)\right)
$$


for $|\alpha| \leqslant 2 m,|\beta| \leqslant m$ and some $k \geqslant[n / 2 m]+3([r]:=\max \{j \in \mathbb{N}: j \leqslant r\})$.

(2) There exist constants $c_{1}, c_{2}>0$ such that

$$
\begin{aligned}
& (-1)^{m} \operatorname{Re} \sum_{|\alpha|=2 m}\left[a_{\alpha}(t, x)+\tilde{a}_{\alpha}(t, x)\right] \xi^{a} \geqslant c_{1}|\xi|^{2 m} \\
& \quad \text { for } \xi \in \mathbb{R}^{n}, t \in[0, T], \quad x \in \bar{\Omega}, \\
& \left\|\left[A(t)-A^{*}(t)\right] \varphi\right\| \leqslant c_{2}\|\varphi\|_{m} \quad \text { for } \varphi \in \dot{H}^{m}(\Omega) \cap H^{2 m}(\Omega), t \in[0, T],
\end{aligned}
$$

where $A^{*}(t)$ denotes the formal adjoint to the operator $A(t)$, and $\|\|,.\|.\|_{m}$ denote the norms in $L_{2}(\Omega)$ and $H^{m}(\Omega)$, respectively.

(3) There exists a constant $c_{3} \geqslant 0$ such that

$$
-\operatorname{Re}\langle B(t) \varphi, \varphi\rangle \leqslant c_{3}\|\varphi\|^{2} \text { for } \varphi \in \stackrel{\circ}{H}^{m}(\Omega), t \in[0, T]
$$

(here $\langle.,$.$\rangle denotes the inner product in L_{2}(\Omega)$ ).

Remarks. (1) We admit that the coefficients of $A$ and $B$ are divided into two different parts, one being continuously differentiable with bounded derivatives, and the other lying in some spatial Sobolev space for every $t \in[0, T]$. This is essential for the application to non-linear problems.

(2) Condition (1.6) means that the part of $A(t)$ containing the derivatives $\partial_{x}^{\alpha} \varphi$ with $m+1 \leqslant|\alpha| \leqslant 2 m$ is symmetric. This condition is also used in [2]. An equivalent formulation of (1.6) is used in [3], (3.5), (3.6).

(3) Condition (1.7) is needed for the energy estimate. In the case $m=1$, if $b_{\beta}+\tilde{b}_{\beta}$ is real valued for $|\beta|=1,(1.7)$ holds automatically if $k \geqslant[n / 2 m]+4$ in (1.4). This can be shown by integrating by parts (compare [9]). More practical conditions for $B(t)$ guaranteeing (1.7) are given in [9].

(4) By Sobolev's lemma it follows from (1.4) and $k \geqslant[n / 2 m]+3$ that $\tilde{a}_{\alpha}(t) \in C_{b}(\bar{\Omega})$ for $t \in[0, T]$. Hence (1.5) is well defined:

The aim of this paper is to prove the existence of a unique solution

$$
u \in \mathscr{C}_{T}^{k}:=\bigcap_{j=0}^{k} C^{j}\left([0, T], H^{(k-j) m}(\Omega)\right)
$$

of (1.1). More precisely, we prove the following theorem.

Theorem 1.1. Let Assumption 1.1 be satisfied for some $k \geqslant k_{0}:=[n / 2 m]+4$ and let $2 \leqslant j \leqslant k$. If

$$
f \in \mathscr{C}_{T}^{j-2} \cap C^{j-1}\left([0, T], L_{2}(\Omega)\right)
$$

and $u^{0} \in H^{j m}(\Omega), u^{1} \in H^{(j-1) m}(\Omega)$ such that $\left(u^{0}, u^{1}, f\right)$ satisfies the compatibility condition (defined in section 2) of order $j$, then (1.1) has a unique solution $u \in \mathscr{C}_{T}^{j}$. Furthermore

$$
\begin{aligned}
|u(t)|_{j}:= & \sum_{v=0}^{j}\left\|\partial_{t}^{v} u(t)\right\|_{(j-v) m} \\
\leqslant & \exp \left(C_{1} t\right)\left(D_{1}|u(0)|_{j}+C_{2} \int_{0}^{t}\left[\left\|\partial_{t}^{j-1} f(\tau)\right\|+|f(\tau)|_{j-2}\right] \mathrm{d} \tau\right) \\
& +D_{2}|f(t)|_{j-2} \text { for } t \in[0, T],
\end{aligned}
$$


where the constants $C_{1}, C_{2}>0$ depend only on $c_{1}, c_{2}, c_{3}$ (of Assumption 1.1), $j$, and

$$
\begin{aligned}
& \sup _{|\alpha| \leqslant 2 m,|\beta| \leqslant m} \sup _{|\gamma|+j m \leqslant(k-1) m} \sup _{[0, T] \times \bar{\Omega}}\left(\left|\partial_{t}^{j} \partial_{x}^{\gamma} a_{\alpha}(t, x)\right|+\left|\partial_{t}^{j} \partial_{x}^{\gamma} b_{\beta}(t, x)\right|\right), \\
& \sup _{|\alpha| \leqslant 2 m,|\beta| \leqslant m} \sup _{[0, T]}\left(\left|\tilde{a}_{\alpha}(t) \tilde{\left.\right|_{k-1}}+\right| \tilde{b}_{\beta}(t) \tilde{\mid}_{k-1}\right)
\end{aligned}
$$

$\left(\left.\left|\tilde{a}_{\alpha}(t) \tilde{\mid}_{k-1}:=\right| \partial_{t} \tilde{a}_{\alpha}(t)\right|_{k-2}+\left\|\tilde{a}_{\alpha}(t)\right\|_{(k-2) m}\right)$, whereas $D_{1}, D_{2}>0$ depend only on $j, c_{1}, c_{2}$, $c_{3},(1.11)$, and

$$
\sup _{|\alpha| \leqslant 2 m .|\beta| \leqslant m} \sup _{[0, T]}\left(\left|\tilde{a}_{\alpha}(t)\right|_{k-2}+\left|\tilde{b}_{\beta}(t)\right|_{k-2}\right) .
$$

Remarks. (1) The fact that $D_{1}, D_{2}$ depend only on (1.13) and not on (1.12) is essential for the iteration procedure in [9], where this theorem is used. If $D_{1}, D_{2}$ are allowed to depend on (1.12), then the condition $k \geqslant[n / 2 m]+4$ in Theorem 1.1 could be relaxed to $k \geqslant[n / 2 m]+3$. But this would require a more complicated proof.

(2) The term $|u(0)|_{j}$ can be estimated by $\left\|u^{0}\right\|_{j m},\left\|u^{1}\right\|_{(j-1) m}$ and $|f(0)|_{j-2}$ (compare (2.8)).

(3) Condition (1.9) can be relaxed to the assumption that $f \in \mathscr{C}_{T}^{j-2}$ and $\partial_{t}^{j-1} f \in L_{2}$ $\left([0, T], L_{2}(\Omega)\right)$. Even (1.4) can be slightly relaxed.

There are many papers dealing with problems of the type (1.1) with $B=0$. We only mention Kato [7], Lions and Magenes [10], and Dafermos and Hrusa [3]. In these papers the existence of the solution is proved in two different ways: by the aid of semigroups ([7] and [10]) and by energy methods ([10], \$3.8.2, and [3]). In the case $m=1$, if

$$
|\operatorname{Re}\langle B(t) \varphi, \varphi\rangle| \leqslant c\|\varphi\|^{2} \quad \text { for } \varphi \in \stackrel{\circ}{H}^{m}(\Omega), t \in[0, T],
$$

Ikawa [5] proved the existence of a solution using semigroups. He considers operators $A(t)$ and $B(t)$ with vanishing $\tilde{a}_{\alpha}$ and $\tilde{b}_{\beta}$. In addition to the Dirichlet boundary condition he studies the Neumann boundary condition. Recently Koch proved in [8] an existence theorem for systems of hyperbolic equations with real-valued coefficients. He assumes that $\Omega$ is bounded and studies the more complicated case of timedependent boundary conditions. Furthermore, he considers a problem similar to (1.1) with $m=1$ (compare (2.12) in [8]). He uses energy methods.

The proof in this paper is also based on energy estimates but differs from the proof in [8]. Section 3 deals with elliptic equations of order $2 m$. In particular, the regularity of a solution of elliptic equations is studied. In section 4 the existence of a unique strong solution $u \in \mathscr{C}_{T}^{2}$ of (1.1) is proved by the method of Faedo-Galerkin, which uses an approximation in finite-dimensional function-spaces. A higher degree of regularity is obtained by induction in section 5 , by solving a system of a simple integral equations and an equation of the type of (1.1) (compare (5.3), (5.4)).

\section{Notation. The compatibility condition}

By $\Omega$ we denote a domain $\Omega \subset \mathbb{R}^{n}$ with $\partial \Omega \in C^{\infty}$ such that $\partial \Omega$ is bounded (or empty) or such that

$$
\Omega=\mathbb{R}^{n^{\prime}} \times \mathbf{\Omega}^{\prime}
$$


with $n^{\prime}<n$ and bounded $\Omega^{\prime} \subset \mathbb{R}^{n-n^{\prime}}$. Let

$$
\partial_{x}^{\alpha} \varphi:=\frac{\partial^{|\alpha|} \varphi}{\partial x_{1}^{\alpha_{1}} \cdots \partial x_{n}^{\alpha_{n}}} \quad \text { for } \alpha \in \mathbb{N}_{0}^{n},
$$

where $|\alpha|=\alpha_{1}+\cdots+\alpha_{n}$. We set

$C_{b}^{k}(\Omega):=\left\{\varphi \in C^{k}(\Omega): \partial_{x}^{\alpha} \varphi\right.$ is bounded in $\Omega$ for $\left.|\alpha| \leqslant k\right\}$.

We use the abbreviation $u^{\prime}=\partial_{t} u, u^{\prime \prime}=\partial_{t}^{2} u$ for time-dependent functions. Furthermore we write $u(t) \in \stackrel{\circ}{H}^{m}(\Omega)$ instead of $u(t,.) \in \stackrel{\circ}{H}^{m}(\Omega)$. Recalling the definition of $\mathscr{C}_{T}^{k}$ in (1.8) we set

$$
|u(t)|_{k}:=\sum_{j=0}^{k}\left\|\partial_{t}^{j} u(t)\right\|_{(k-j) m} \text { for } t \in[0, T]
$$

for $u \in \mathscr{C}_{T}^{k}$. Besides $\mathscr{C}_{T}^{k}$ we use the linear space

$$
\tilde{\mathscr{C}}_{T}^{k}:=\bigcap_{j=1}^{k} C^{j}\left([0, T], H^{(k-j) m}(\Omega)\right) \text {. }
$$

Note that $C\left([0, T], H^{k m}(\Omega)\right)$ contains $\mathscr{C}_{T}^{k}$ but not $\tilde{\mathscr{C}}_{T}^{k}$. For $v \in \tilde{\mathscr{C}}_{T}^{k}$ we set

$$
\begin{aligned}
|v(t)|_{k} & :=\sum_{j=1}^{k}\left\|\partial_{t}^{j} v(t)\right\|_{(k-j) m}+\|v(t)\|_{(k-1) m} \\
& =\left|v^{\prime}(t)\right|_{k-1}+\|v(t)\|_{(k-1) m} \text { for } t \in[0, T] .
\end{aligned}
$$

In order to give the compatibility condition we assume that $u \in \mathscr{C}_{T}^{k}$ is a solution of (1.1). From $u(t) \in \dot{H}^{m}(\Omega)$ for $t \in[0, T]$ it follows that $\partial_{i}^{j} u(0) \in \mathscr{H}^{m}(\Omega)$ for $j=0, \ldots$, $k-1$ (compare [9]). Differentiating (1.1) formally $(j-2)$-times, we obtain

$$
\begin{aligned}
\partial_{t}^{j} u(0)= & \partial_{t}^{j-2} f(0)-\sum_{v=0}^{j-2}\left(\begin{array}{c}
j-2 \\
v
\end{array}\right)\left\{\left[\partial_{t}^{v} A(0)\right] \partial_{t}^{j-2-v} u(0)\right. \\
& \left.+\left[\partial_{t}^{v} B(0)\right] \partial_{t}^{j-1}-v u(0)\right\}
\end{aligned}
$$

where

$$
\partial_{t}^{v} A(0)=\sum_{|\alpha| \leqslant 2 m}\left[\partial_{t}^{v} a_{\alpha}(0, .)+\partial_{t}^{v} \tilde{a}_{x}(0, .)\right] \partial_{x}^{\alpha}
$$

and $\partial_{t}^{v} B(0)$ is given analogously. We make the following definition.

Definition 2.1. We say that $\left(u^{0}, u^{1}, f\right)$ satisfies the compatibility condition of order $k \in \mathbb{N}$ if $u^{j} \in \dot{H}^{m}(\Omega)$ for $j=0, \ldots, k-1$, where $u^{j}$ is recursively defined by

$$
u^{j}:=\partial_{t}^{j-2} f(0)-\sum_{v=0}^{j-2}\left(\begin{array}{c}
j-2 \\
v
\end{array}\right)\left\{\left[\partial_{t}^{v} A(0)\right] u^{j-2-v}+\left[\partial_{t}^{v} B(0)\right] u^{j-1-v}\right\}
$$

for $j \geqslant 2$.

Remark. Let Assumption 1.1 be satisfied for some $k \geqslant[n / 2 m]+3$ and let $2 \leqslant j \leqslant k$. If

$$
u^{0} \in H^{j m}(\Omega), u^{1} \in H^{(j-1) m}(\Omega), \partial_{t}^{v-2} f(0) \in H^{(j-v) m}(\Omega) \text { for } v=2, \ldots, j,
$$

then $u^{v}$ is well defined by (2.7) for $v=2, \ldots, j$. Furthermore, it follows from Lemma 
8.2 of [9] and induction that

$$
|u(0)|_{j}=\sum_{v=0}^{j}\left\|u^{v}\right\|_{(j-v) m} \leqslant D\left[\left\|u^{0}\right\|_{j m}+\left\|u^{1}\right\|_{(j-1) m}+|f(0)|_{j-2}\right],
$$

where $D>0$ depends only on $j,(1.11)$ and (1.13).

\section{Elliptic equations}

Consider the elliptic differential equations

$$
A(t) u(t)=f(t) \text { for } t \in[0, T],
$$

where $A$ satisfies Assumption 1.1 for some $k \geqslant k_{0}=[n / 2 m]+4$. We choose

$$
\left.\begin{array}{l}
\mathcal{c}_{\alpha \beta} \in C_{b}^{(k-2) m+|\alpha|}([0, T] \times \bar{\Omega}), \\
\tilde{c}_{\alpha \beta} \in C\left([0, T], H^{(k-3) m+|\alpha|}(\Omega)\right)
\end{array}\right\}
$$

such that

$$
A(t) u(t)=\sum_{|\alpha|,|\beta| \leqslant m}(-1)^{|\alpha|} \partial_{x}^{\alpha}\left\{\left[c_{\alpha \beta}(t)+\tilde{c}_{\alpha \beta}(t)\right] \partial_{x}^{\beta} u(t)\right\}
$$

(compare, e.g., [1], section 8). It follows from (1.5) that

$$
(-1)^{m} \operatorname{Re} \sum_{|\alpha|=|\beta|=m}\left[c_{\alpha \beta}(t, x)+\tilde{c}_{\alpha \beta}(t, x)\right] \xi^{\alpha+\beta} \geqslant c_{1}|\xi|^{2 m}
$$

for $\xi \in \mathbb{R}^{n}, t \in[0, T]$ and $x \in \bar{\Omega}$. We set

$$
\begin{aligned}
a(t, \varphi, \psi):= & \sum_{|\alpha|=|\beta| \leqslant m}\left\langle\left[c_{\alpha \beta}(t)+\tilde{c}_{\alpha \beta}(t)\right] \partial_{x}^{\beta} \varphi, \partial_{x}^{\alpha} \psi\right\rangle \\
& \text { for } \varphi, \psi \in H^{m}(\Omega), t \in[0, T]
\end{aligned}
$$

and study instead of (3.1) the equation

$$
a(t, u(t), \varphi)=\langle f(t), \varphi\rangle \text { for every } \varphi \in C_{0}^{\infty}(\Omega), t \in[0, T] .
$$

We prove the following lemma.

Lemma 3.1. Let $k \geqslant[n / 2 m]+4$ and let Assumption 1.1 be satisfied. If $f \in C([0, T]$, $\left.H^{j}(\Omega)\right)$ for some $j \leqslant(k-2) m$ and if $u \in C\left([0, T], \stackrel{\circ}{H}^{m}(\Omega)\right)$ such that (3.6) holds, then $u \in C\left([0, T], H^{j+2 m}(\Omega)\right)$. Furthermore

$$
\|u(t)\|_{j+2 m} \leqslant d\left(\|f(t)\|_{j}+\|u(t)\|\right) \text { for } t \in[0, T],
$$

where $d>0$ depends only on $j$ and on

$$
\sup _{|\alpha| \leqslant 2 m} \sup _{\{0, T]}\left(\sup _{|\gamma| \leqslant(k-2) m} \sup _{x \in \Omega}\left|\partial_{x}^{\gamma} a_{\alpha}(t, x)\right|+\left\|\tilde{a}_{\alpha}(t)\right\|_{(k-2) m}\right)
$$

(and on $\Omega$ ).

Proof. Let $\Omega$ be of the form (2.1). In the other cases Lemma 3.1 can be shown 
analogously. At first we prove the assertion in the case $j=0$. We set

$$
\Omega_{R}:=\{x \in \Omega:|x|<R\}
$$

and choose $R>0$ so large that

Let

$$
\left\{x \in \Omega=\mathbb{R}^{n^{\prime}} \times \Omega^{\prime}:\left|x_{i}\right| \leqslant 1 \quad \text { for } i=1, \ldots, n^{\prime}\right\} \subset \Omega_{R} .
$$

$$
T_{z}\left(\Omega_{R}\right):=\left\{x+z: x \in \Omega_{R}\right\} \quad \text { for } z=\left(z_{1}, \ldots, z_{n^{\prime}}, 0, \ldots, 0\right) .
$$

Note that $(k-3) m \geqslant[n / 2]+1$ and that therefore

$$
c_{\alpha \beta}(t)+\tilde{c}_{\alpha \beta}(t) \in C_{b}^{|\alpha|}(\bar{\Omega}) \quad \text { for }|\alpha|,|\beta| \leqslant m, t \in[0, T]
$$

by the lemma of Sobolev. Hence $a(t, \varphi, \psi)$ is right $m$-smooth for every $t \in[0, T]$ in the sense of Definition 9.1 of [1]. By the proof of Theorem 9.8 in [1] it follows from (3.6) that

$$
\|u(t)\|_{H^{2 m}\left(T_{z}\left(\Omega_{R}\right)\right)} \leqslant d_{1}\left(\|f(t)\|_{L_{2}\left(T_{z}\left(\Omega_{R+1}\right)\right)}+\|u(t)\|_{H^{m}\left(T_{z}\left(\Omega_{R+1}\right)\right)}\right) .
$$

for every $z \in S:=\mathbb{Z}^{n^{\prime}} \times\{(0, \ldots, 0)\}$, where $d_{1}>0$ can be chosen to be independent of $z$. Note that

$$
\Omega=\bigcup_{z \in S} T_{z}\left(\Omega_{R}\right)=\bigcup_{z \in S} T_{z}\left(\Omega_{R+1}\right),
$$

and that every $x \in \Omega$ is contained only in a fixed finite number of sets $T_{z}\left(\Omega_{R+1}\right)$. Hence summation over all $z \in S$ yields $u(t) \in H^{2 m}(\Omega)$ and

$$
\|u(t)\|_{2 m} \leqslant d_{2}\left(\|f(t)\|+\|u(t)\|_{m}\right)
$$

for $t \in[0, T]$. In view of (3.4) there exist constants $d_{3}, d_{4}>0$ such that

$$
\operatorname{Re} a(t, \varphi, \varphi) \geqslant d_{3}\|\varphi\|_{m}^{2}-d_{4}\|\varphi\|^{2} \quad \text { for } \varphi \in \stackrel{\circ}{H}^{m}(\Omega), t \in[0, T]
$$

Hence it follows from (3.6) (with $\varphi:=u(t)$ ) and (3.13) that (3.7) holds in the case $j=0$. In particular, we have that $\|u(t)\|_{2 m}$ is bounded on $[0, T]$.

If $t, t_{1} \in[0, T]$, we obtain from (3.6) that

with

$$
a\left(t, u(t)-u\left(t_{1}\right), \varphi\right)=\left\langle g_{1}\left(t, t_{1}^{\prime}\right), \varphi\right\rangle \text { for every } \varphi \in C_{0}^{\infty}(\Omega), t \in[0, T]
$$

$$
g_{1}\left(t, t_{1}\right):=f(t)-f\left(t_{1}\right)+\left[A\left(t_{1}\right)-A(t)\right] u\left(t_{1}\right) .
$$

With Lemma 8.2 in [9] we conclude that $\left\|g_{1}\left(t, t_{1}\right)\right\| \rightarrow 0$ as $t \rightarrow t_{1}$. Hence (3.7) applied to $(3.15)$ yields $\left\|u(t)-u\left(t_{1}\right)\right\|_{2 m} \rightarrow 0$ as $t \rightarrow t_{1}$ and therefore $u \in C\left([0, T], H^{2 m}(\Omega)\right)$. This proves the assertion for $j=0$.

Now let Lemma 3.1 be proved for $j=0, \ldots, J \leqslant(k-2) m-1$. By the induction hypothesis we have $u \in C\left([0, T], H^{J+2 m}(\Omega)\right)$ and (3.7) with $j=J$. Let $\psi \in C_{0}^{\infty}(\Omega)$. From (3.6) and (3.3) we conclude that

with

$$
a(t, \psi u(t), \varphi)=\left\langle g_{2}(t), \varphi\right\rangle \text { for } \varphi \in C_{0}^{\infty}(\Omega), t \in[0, T]
$$

$$
g_{2}(t):=\psi f(t)+A(t)(\psi u(t))-\psi A(t) u(t)
$$

Note that the derivatives of $u$ of order $2 m$ vanish on the right-hand side of (3.18). From $\left.\tilde{a}_{\alpha} \in C(0, T], H^{(k-2) m}(\Omega)\right)$ for $|\alpha| \leqslant 2 m$ and Lemma 8.2 in [9] it follows that $g_{2} \in$ 
$C\left([0, T], H^{J+1}(\Omega)\right)$. Setting $\varphi=\partial \Phi / \partial x_{i}$ in $(3,17)$ with $\Phi \in C_{0}^{\infty}(\Omega)$, we conclude that

$$
a\left(t, \frac{\partial(\psi u(t))}{\partial x_{i}}, \Phi\right)=\left\langle g_{3}(t), \Phi\right\rangle \text { for } \Phi \in C_{0}^{\infty}(\Omega), t \in[0, T]
$$

where

$$
g_{3}(t):=\frac{\partial g_{2}(t)}{\partial x_{i}}-\sum_{|\alpha| \leqslant 2 m}\left(\frac{\partial}{\partial x_{i}}\left[a_{\alpha}(t)+\tilde{a}_{\alpha}(t)\right]\right) \partial_{x}^{\alpha}(\psi u(t)) .
$$

We apply (3.7) with $j=J$ to (3.19) and obtain

$$
\begin{aligned}
\left\|\frac{\partial \psi u(t)}{\partial x_{i}}\right\|_{J+2 m} & \leqslant d\left(\left\|g_{3}(t)\right\|_{J}+\|\psi u(t)\|\right) \\
& \leqslant d_{1}\left(\left\|\frac{\partial(\psi f(t))}{\partial x_{i}}\right\|_{J}+\|u(t)\|_{\left.H^{J+2 m(\operatorname{supp} \psi)}\right)}\right)
\end{aligned}
$$

for $t \in[0, T]$ and $i=1, \ldots, n$, where $d_{1}>0$ depends only on (3.8), $J$ and $\psi$.

An analogous procedure can be performed around the boundary points. In fact, let $x_{0} \in \partial \Omega$ and let $U$ be an open neighbourhood of $x_{0}$ such that there exists a $C^{\infty}$ mapping transforming $U \cap \Omega$ in $K_{R}^{+}:=\left\{x \in \mathbb{R}^{n}:|x|<R, x_{n}>0\right\}$ and $\bar{U} \cap \partial \Omega$ in $\Gamma_{R}:=\left\{x \in \mathbb{R}^{n}:|x| \leqslant R, x_{n}=0\right\}$. Since such a mapping preserves the properties of our elliptic equation; we can assume that $U \cap \Omega=K_{R}^{+}, \bar{U} \cap \partial \Omega=\Gamma_{R}$.

For given $R^{\prime} \in(0, R)$ we choose $\psi \in C_{0}^{\infty}\left(K_{R}\right)$ with $\psi=1$ on $K_{R^{\prime}}:=\left\{x \in \mathbb{R}^{n}:|x|<R^{\prime}\right\}$. Then (3.21) follows as above for $i=1, \ldots, n-1$, since $\partial(\psi u(t)) / \partial x_{i} \in \dot{H}^{m}\left(K_{R}^{+}\right)$for $i=1, \ldots, n-1$.

Since $u \in H^{2 m}(\Omega)$, we conclude from (3.17) that

$$
A(t)[\psi u(t)]=g_{2}(t) \text { for } t \in[0, T]
$$

and hence

$$
\left[a_{(0, \ldots, 0,2 m)}(t)+\tilde{a}_{(0, \ldots, 0,2 m)}(t)\right] \partial_{x}^{(0, \ldots, 0,2 m)}(\psi u(t))=g_{4}(t)
$$

with a suitable $g_{4}$, where according to (3.18) and (3.21)

$$
\left\|g_{4}(t)\right\|_{J+1} \leqslant d_{2}\left[\|\psi f(t)\|_{J+1}+\|u(t)\|_{H^{J+2 m}(\operatorname{supp} \psi)}\right] .
$$

Since

$$
\left|a_{(0, \ldots, 0,2 m)}(t, x)+\tilde{a}_{(0, \ldots, 0,2 m)}(t, x)\right| \geqslant c_{1}>0
$$

by (1.5), we conclude from (3.22) and (1.4) that

$$
\mid \partial_{x}^{(0, \ldots, 0,2 m)} \psi u(t) \|_{J+1} \leqslant d_{3}\left(\|\psi f(t)\|_{J+1}+\|u(t)\|_{H^{J+2 m}(\text { supp } \psi)}\right) .
$$

We choose a finite number of suitable functions $\psi_{1}, \ldots, \psi_{v}$. Then we conclude from (3.21) and (3.23) that

$$
\|u(t)\|_{H^{j+1+2 m}\left(T_{z}\left(\Omega_{R}\right)\right)} \leqslant d_{4}\left(\|f(t)\|_{H^{j+1}\left(T_{z}\left(\Omega_{R+1}\right)\right)}+\|u(t)\|_{H^{j+2 m}\left(T_{z}\left(\Omega_{R+1}\right)\right)}\right)
$$

for $t \in[0, T]$ and $z=(0, \ldots, 0)$, where $d_{4}>0$ depends only on (3.8) and $J$. Note that we can prove (3.24) for arbitrary $z \in S=\mathbb{Z}^{n} \times\{(0, \ldots, 0)\}$ by the same argument using the functions $\psi_{1}(x-z), \ldots, \psi_{v}(x-z)$. Hence $d_{4}$ can be chosen such that (3.24) holds for every $z \in S$. Using the argument leading to (3.13) we conclude with the induction hypothesis that (3.7) holds for $j=J+1$. Finally, $u \in C\left([0, T], H^{J+1+2 m}(\Omega)\right)$ follows 
applying (3.7) with $j=J+1$ to (3.15), since $\left\|g_{1}\left(t, t_{1}\right)\right\|_{J+1} \rightarrow 0$ as $t \rightarrow t_{1}$ by Lemma 8.2 of [9].

\section{Existence and uniqueness of the solution}

In this section we suppose that Assumption 1.1 is satisfied for $k=k_{0}=[n / 2 m]+4$. Let $a(t, \varphi, \psi)$ be defined by (3.2)-(3.5). Note that (1.6) implies that we can choose the coefficients of $a(t, \varphi, \psi)$ such that

$$
c_{\alpha \beta}+\tilde{c}_{\alpha \beta}=\overline{\left(c_{\beta \alpha}+\tilde{c}_{\beta \alpha}\right)} \text { for } m+1 \leqslant|\alpha|+|\beta| \leqslant 2 m .
$$

Furthermore it follows from (1.5) that there exist constants $d, d_{1}>0$ such that for

$$
a_{d}(t, \varphi, \psi):=\sum_{\substack{|\alpha|,|\beta| \leqslant m \\|\alpha|+|\beta| \geqslant m+1}}\left\langle\left[c_{\alpha \beta}(t)+\tilde{c}_{\alpha \beta}(t)\right] \partial_{x}^{\beta} \varphi, \partial_{x}^{\alpha} \psi\right\rangle+d\langle\varphi, \psi\rangle
$$

it holds that

$$
a_{d}(t, \varphi, \varphi)=\operatorname{Re} a_{d}(t, \varphi, \varphi) \geqslant d_{1}\|\varphi\|_{m}^{2} \text { for } \varphi \in \check{H}^{m}(\Omega), t \in[0, T] .
$$

Here and in the following we denote by $d, d_{1}, d_{2}, \ldots$ positive constants depending only on $c_{1}, c_{2}, c_{3}$ (of Assumption 1.1) and on (1.11), (1.13).

In order to prove the existence of a solution of (1.1) we use the method of Faedo-Galerkin and follow the considerations in [3]. We suppose that

$$
u^{0} \in \stackrel{\circ}{H}^{m}(\Omega) \cap H^{2 m}(\Omega), \quad u^{1} \in \stackrel{\circ}{H}^{m}(\Omega), \quad f \in H^{1}\left([0, T], L_{2}(\Omega)\right)
$$

where the last condition means that $f, f^{\prime} \in L_{2}\left([0, T], L_{2}(\Omega)\right)$. Note that this implies that $f \in C\left([0, T], L_{2}(\Omega)\right)$. Let

$$
\left\{\xi_{1}, \xi_{2}, \ldots\right\} \subset \dot{H}^{m}(\Omega) \cap H^{2 m}(\Omega)
$$

such that every finite subset is linearly independent and $\operatorname{span}\left\{\xi_{1}, \xi_{2}, \ldots\right\}$ is dense in $L_{2}(\Omega)$. We seek an approximate solution

of

$$
u_{j}(t)=\sum_{v=1}^{j} u_{j v}(t) \xi_{v}
$$

$$
\left.\begin{array}{c}
\left\langle u_{j}^{\prime \prime}(t)+A(t) u_{j}(t)+B(t) u_{j}^{\prime}(t)-f(t), \xi_{v}\right\rangle=0 \text { for } t \in[0, T], v=1, \ldots, j, \\
u_{j}(0)=u_{j}^{0}, \quad u_{j}^{\prime}(0)=u_{j}^{\mathrm{t}},
\end{array}\right\}
$$

where $u_{j}^{0}, u_{j}^{1} \in \operatorname{span}\left\{\xi_{1}, \ldots, \xi_{j}\right\}$ are chosen so that $\left\|u_{j}^{v}-u^{v}\right\|_{(2-v) m} \rightarrow 0$ as $j \rightarrow \infty$ $(v=0,1)$. Since $(4.6)$ is a system of ordinary differential equations for the coefficients $u_{j v}(v=1, \ldots, j)$, it follows from standard classical theory that a solution $\left(u_{j 1}, \ldots, u_{j j}\right) \in C^{2}([0, T])$ exists. From (4.6) we conclude that $\left(u_{j 1}, \ldots, u_{j j}\right)$ $\in H^{3}([0, T])$. Hence $u_{j} \in H^{3}\left([0, T], H^{2 m}(\Omega)\right)$.

We set

$$
\left|u_{j}(t)\right|_{E}:=\left[a_{d}\left(t, u_{j}(t), u_{j}(t)\right)+\left\|u_{j}^{\prime}(t)\right\|^{2}\right]^{1 / 2} \text { for } t \in[0, T]
$$


It holds that

$$
\frac{\mathrm{d}}{\mathrm{d} t}\left|u_{j}(t)\right|_{E}^{2}=2 \operatorname{Re}\left[a_{d}\left(t, u_{j}(t), u_{j}^{\prime}(t)\right)+\left\langle u_{j}^{\prime \prime}(t), u_{j}^{\prime}(t)\right\rangle\right]+a_{d}^{\prime}\left(t, u_{j}(t), u_{j}(t)\right)
$$

for $t \in[0, T]$, where

$$
a_{d}^{\prime}(t, \varphi, \psi)=\sum_{m+1 \leqslant|\alpha|+|\beta| \leqslant 2 m}\left\langle\left[c_{\alpha \beta}^{\prime}(t)+\tilde{c}_{\alpha \beta}^{\prime}(t)\right] \partial_{x}^{\beta} \varphi, \partial_{x}^{\alpha} \psi\right\rangle .
$$

Replacing $\xi_{v}$ in (4.6) by $u_{j}^{\prime}(t) \in \operatorname{span}\left\{\xi_{1}, \ldots, \xi_{j}\right\}$, we conclude that

$$
\begin{aligned}
& \frac{\mathrm{d}}{\mathrm{d} t}\left|u_{j}(t)\right|_{E}^{2} \\
& =2 \operatorname{Re}\left\langle f(t)-B(t) u_{j}^{\prime}(t)-\sum_{|\alpha|+|\beta| \leqslant m} \partial_{x}^{\alpha}\left\{\left[c_{\alpha \beta}(t)+\tilde{c}_{\alpha \beta}(t)\right] \partial_{x}^{\beta} u_{j}(t)\right\}+d u_{j}(t), u_{j}^{\prime}(t)\right\rangle \\
& \quad+a_{d}^{\prime}\left(t, u_{j}(t), u_{j}(t)\right) .
\end{aligned}
$$

With (1.7) and (4.3) we obtain

$$
\frac{\mathrm{d}}{\mathrm{d} t}\left|u_{j}(t)\right|_{E}^{2} \leqslant 2\left[\|f(t)\|+d_{2}\left|u_{j}(t)\right|_{E}\right]\left|u_{j}(t)\right|_{E}
$$

and

$$
\frac{\mathrm{d}}{\mathrm{d} t}\left|u_{j}(t)\right|_{E} \leqslant\|f(t)\|+d_{2}\left|u_{j}(t)\right|_{E} \quad \text { for } t \in[0, T] .
$$

With Gronwall's Lemma it follows that there is a constant $C>0$ such that $\left|u_{j}(t)\right|_{E} \leqslant C$ for $t \in[0, T]$ and $j \in \mathbb{N}$.

In a similar way we prove that $\left|u_{j}^{\prime}(t)\right|_{E}$ is bounded. We differentiate (4.6) with respect to $t$ and obtain

$$
\left\langle u_{j}^{\prime \prime \prime}(t)+A(t) u_{j}^{\prime}(t)+B(t) u_{j}^{\prime \prime}(t)+A^{\prime}(t) u_{j}(t)+B^{\prime}(t) u_{j}^{\prime}(t)-f^{\prime}(t), \xi_{v}\right\rangle=0
$$

for $t \in[0, T], v=1, \ldots, j$. We replace $\xi_{v}$ by $u_{j}^{\prime \prime}(t)$ and conclude by the same argument as above that

with

$$
\frac{\mathrm{d}}{\mathrm{d} t}\left|u_{j}^{\prime}(t)\right|_{E}^{2}=-\left\langle A^{\prime}(t) u_{j}(t), u_{j}^{\prime \prime}(t)\right\rangle+r(t)
$$

$$
|r(t)| \leqslant 2\left\|f^{\prime}(t)\right\|\left|u_{j}^{\prime}(t)\right|_{E}+d_{3}\left|u_{j}^{\prime}(t)\right|_{E}^{2} \leqslant\left\|f^{\prime}(t)\right\|^{2}+\left(d_{3}+1\right)\left|u_{j}^{\prime}(t)\right|_{E}^{2}
$$

a.e. in $[0, T]$. Let $t_{0} \in[0, T]$. We use

$$
\int_{0}^{t}\left\langle A^{\prime}(\tau) u_{j}(\tau), u_{j}^{\prime \prime}(\tau)\right\rangle \mathrm{d} \tau=\left.\left\langle A^{\prime}(\tau) u_{j}(\tau), u_{j}^{\prime}(\tau)\right\rangle\right|_{\tau} ^{t}=0-\int_{0}^{t}\left\langle\partial_{\tau}\left(A^{\prime}(\tau) u_{j}(\tau)\right), u_{j}^{\prime}(\tau)\right\rangle \mathrm{d} \tau
$$

and

$$
\begin{aligned}
& \left|\left\langle A^{\prime}(t) u_{j}(t), u_{j}^{\prime}(t)\right\rangle\right|=\mid a^{\prime}\left(t, u_{j}(t),\left.u_{j}^{\prime}(t)|\leqslant \varepsilon| u_{j}^{\prime}(t)\right|_{E} ^{2}+\frac{d_{4}}{\varepsilon}\left|u_{j}(t)\right|_{E}^{2},\right. \\
& \left\langle\partial_{\varepsilon}\left(A^{\prime}(\tau) u_{j}(\tau)\right), u_{j}^{\prime}(\tau)\right\rangle \leqslant d_{1}^{\prime}\left(\left\|u_{j}(\tau)\right\|_{m}^{2}+\left\|u_{j}^{\prime}(\tau)\right\|_{m}^{2}\right)
\end{aligned}
$$

with $\varepsilon>0$. Here and in the following we denote by $d_{1}^{\prime}, d_{2}^{\prime}, \ldots$ positive constants 
depending on $c_{1}, c_{2}$ and $c_{3}$, and on (1.11) and (1.12). Integration of (4.12) yields

$$
\begin{aligned}
\left|u_{j}^{\prime}(t)\right|_{E}^{2} \leqslant & d_{5}\left[\left|u_{j}^{\prime}(0)\right|_{E}^{2}+\left|u_{j}(0)\right|_{E}^{2}\right]+\varepsilon\left|u_{j}^{\prime}(t)\right|_{E}^{2}+\frac{d_{4}}{\varepsilon}\left|u_{j}(t)\right|_{E}^{2} \\
& +d_{2}^{\prime} \int_{0}^{t}\left[\left|u_{j}^{\prime}(\tau)\right|_{E}^{2}+\left|u_{j}(\tau)\right|_{E}^{2}+\left\|f^{\prime}(\tau)\right\|^{2}\right] \mathrm{d} \tau
\end{aligned}
$$

Note that $\left|u_{j}^{\prime}(0)\right|_{E}^{2}$ is bounded as $j \rightarrow \infty$, since $\left\|\partial_{t}^{v} u_{j}(0)-u^{v}\right\|_{(2-v) m} \rightarrow 0$ as $j \rightarrow \infty$ for $v=0,1,2$, where $u^{2}=f(0)-A(0) u^{0}-B(0) u^{1}$. In fact, (4.6) implies that $u_{j}^{\prime \prime}(0)$ converges weakly to $u^{2}$ in $L_{2}(\Omega)$ and

$$
\varlimsup_{j \rightarrow \infty}\left\|u_{j}^{\prime \prime}(0)\right\|^{2}=\varlimsup_{j \rightarrow \infty}\left\langle f(0)-A(0) u_{j}^{0}-B(0) u_{j}^{1}, u_{j}^{\prime \prime}(0)\right\rangle \leqslant\left\|u^{2}\right\| \varlimsup_{j \rightarrow \infty}\left\|u_{j}^{\prime \prime}(0)\right\| .
$$

Hence $\varlimsup_{j \rightarrow \infty}\left\|u_{j}^{\prime \prime}(0)\right\| \leqslant\left\|u^{2}\right\|$ and it follows that $u_{j}^{\prime \prime}(0) \rightarrow u^{2}$ in $L_{2}(\Omega)$ as $j \rightarrow \infty$.

With Gronwall's lemma we conclude from (4.14) and the boundedness of $\left|u_{j}(t)\right|_{E}^{2}$ that there exists a $C>0$ such that $\left|u_{j}^{\prime}(t)\right|_{E}^{2} \leqslant C$ for $t \in[0, T], j \in \mathbb{N}$. Hence we can extract a subsequence converging weakly to a

$$
u \in H^{2}\left([0, T], L_{2}(\Omega)\right) \cap H^{1}\left([0, T], \stackrel{\circ}{H}^{m}(\Omega)\right) .
$$

By a standard argument (compare $\S 3.8 .2$ in [10]) it follows that

$$
\begin{array}{ll}
u^{\prime \prime}(t)+A(t) u(t)+B(t) u^{\prime}(t)=f(t) & \text { a.e. in }[0, T], \\
u(t) \in \stackrel{\circ}{H}^{m}(\Omega) & \text { for } t \in[0, T], \\
u(0)=u^{0}, \quad u^{\prime}(0)=u^{1} . &
\end{array}
$$

From (4.15) we obtain by the argument leading to (4.10) that

$$
\frac{\mathrm{d}}{\mathrm{d} t}|u(t)|_{E} \leqslant\|f(t)\|+d_{2}|u(t)|_{E} \quad \text { a.e. in }[0, T] \text {. }
$$

Using Gronwall's lemma we conclude that

$$
|u(t)|_{E} \leqslant \mathrm{e}^{d_{2 t}}\left(|u(0)|_{E}+\int_{0}^{t}\|f(\tau)\| \mathrm{d} \tau\right) \text { for } t \in[0, T],
$$

and with (4.3) and (4.7) we obtain

$$
|u(t)|_{1} \leqslant d_{5} \mathrm{e}^{d_{2 t} t}\left(|u(0)|_{1}+\int_{0}^{t}\|f(\tau)\| \mathrm{d} \tau\right) \text { for } t \in[0, T] .
$$

In the rest of this section we prove that $u \in \mathscr{C}_{T}^{2}$ and derive an estimate for $|u(t)|_{2}$. To this end we introduce a convenient concept of a weak solution.

Definition 4.1. Let $u^{0} \in \stackrel{\circ}{H}^{m}(\Omega), u^{1} \in L_{2}(\Omega)$ and $f \in L_{1}\left([0, T], L_{2}(\Omega)\right)$. We say that $u \in H^{1}\left([0, T], L_{2}(\Omega)\right) \cap L_{2}\left([0, T], \stackrel{\circ}{H}^{m}(\Omega)\right)$

is $a$ weak solution of (1.1) if $u(0)=u^{0}$ and

$$
\begin{aligned}
& \int_{0}^{T}\left[-a(t, u(t), v(t))+\left\langle B(t) u(t)+u^{\prime}(t), v^{\prime}(t)\right\rangle+\left\langle B^{\prime}(t) u(t)+f(t), v(t)\right\rangle\right] \mathrm{d} t \\
& =\left\langle u^{1}+B u^{0}, v(0)\right\rangle
\end{aligned}
$$


for every $v \in H^{1}\left([0, T], L_{2}(\Omega)\right) \cap L_{2}\left([0, T], \stackrel{\circ}{H}^{m}(\Omega)\right)$ with $v(T)=0$ (note that a $(t, u(t)$, $v(t))$ is defined by (3.5)).

Remarks. (1) Since $\partial_{1} v \in L_{2}\left([0, T], L_{2}(\Omega)\right)$ it holds $v \in C\left([0, T], L_{2}(\Omega)\right)$. Hence $v(0)$, $v(T)$ are well defined.

(2) Integration by parts shows that $u \in \mathscr{C}_{T}^{2}$ is a solution of (1.1) if and only if it is weak solution.

In a first step we prove the uniqueness of a weak solution.

Lemma 4.1. Let Assumption 1.1 be satisfied for $k=k_{0}$. If $u^{0}=u^{1}=0$ and $f(t)=0$ on $[0, T]$, and if

$$
u \in H^{1}\left([0, T], L_{2}(\Omega)\right) \cap L_{2}\left([0, T], \dot{H}^{m}(\Omega)\right)
$$

is a weak solution of $(1.1)$, then $u(t)=0$ on $[0, T]$.

Proof. We proceed analogously to the proof of the uniqueness in $\$ 3.8 .2$ of [10]. Let $s \in(0, T)$ and

Note that

$$
v(t):= \begin{cases}-\int_{t}^{s} u(\sigma) \mathrm{d} \sigma & \text { for } t<s \\ 0 & \text { for } t \geqslant s .\end{cases}
$$

$$
\begin{aligned}
& \frac{\mathrm{d}}{\mathrm{d} t}\left[a_{d}(t, v(t), v(t))+2 \operatorname{Re}\left\langle B^{\prime}(t) v(t), v(t)\right\rangle+\|u(t)\|^{2}\right] \\
& =2 \operatorname{Re}\left[a_{d}(t, u(t), v(t))+\left\langle B^{\prime}(t) u(t), v(t)\right\rangle+\left\langle u^{\prime}(t), v^{\prime}(t)\right\rangle\right]+r(t)
\end{aligned}
$$

with

$$
|r(t)| \leqslant d_{3}^{\prime}\left[\|v(t)\|_{m}^{2}+\|u(t)\|^{2}\right]
$$

a.e. in $[0, s)$. We conclude from (4.19) that

$$
a_{d}(0, v(0), v(0))+2 \operatorname{Re}\left\langle B^{\prime}(0) v(0), v(0)\right\rangle+\|u(s)\|^{2} \leqslant d_{4}^{\prime} \int_{0}^{s}\left[\|v(t)\|_{m}^{2}+\|u(t)\|^{2}\right] \mathrm{d} t
$$

There exists a $d_{5}^{\prime}>0$ such that

$$
a_{d}(0, v(0), v(0))+2 \operatorname{Re}\left\langle B^{\prime}(0) v(0), v(0)\right\rangle \geqslant \frac{d_{1}}{2}\|v(0)\|_{m}^{2}-d_{5}^{\prime}\|v(0)\|^{2} .
$$

Hence we obtain from (4.21) with the argument proving the uniqueness in $\$ 3.8 .2$ of [10] that $u(t)=0$ for $t \in[0, T]$.

In the next step we prove the existence of a weak solution $u \in \mathscr{C}_{T}^{1}$ of (1.1).

Lemma 4.2. Let Assumption 1.1 be satisfied for $k=k_{0}$. Furthermore let $u^{0} \in \dot{H}^{m}(\Omega)$, $u^{1} \in L_{2}(\Omega)$ and $f \in L_{1}\left([0, T], L_{2}(\Omega)\right)$. Then there exists a weak solution $u \in \mathscr{C}_{T}^{1}$ of $(1.1)$.

Proof. We approximate $u^{0}, u^{1}$ and $f$ by sequences $\left\{u_{j}^{0}\right\}$ in $H^{2 m}(\Omega) \cap \dot{H}^{m}(\Omega),\left\{u_{j}^{1}\right\}$ in $\stackrel{\circ}{H}^{m}(\Omega)$ and $\left\{f_{j}\right\}$ in $H^{1}\left([0, T], L_{2}(\Omega)\right)$ such that $\left\|u^{v}-u_{j}^{v}\right\|_{(1-v) m} \rightarrow 0$ as $j \rightarrow \infty$ 
$(v=0,1)$ and

$$
\int_{0}^{T}\left\|f(\tau)-f_{j}(\tau)\right\| \mathrm{d} \tau \rightarrow 0 \quad \text { as } j \rightarrow \infty .
$$

Let $u_{j} \in H^{2}\left([0, T], L_{2}(\Omega)\right) \cap H^{1}\left([0, T], \dot{H}^{m}(\Omega)\right) \subset \mathscr{C}_{T}^{1}$ be the solution of (4.15) with data $u_{j}^{0}, u_{j}^{1}$ and $f_{j}$, which we have constructed above. We apply (4.18) to

$$
\begin{aligned}
& u_{j}^{\prime \prime}(t)-u_{i}^{\prime \prime}(t)+A(t)\left[u_{j}(t)-u_{\ell}(t)\right]+B(t)\left[u_{j}^{\prime}(t)-u_{\ell}^{\prime}(t)\right]=f_{j}(t)-f_{\ell}(t) \\
& \quad \text { a.e. in }[0, T]
\end{aligned}
$$

and obtain that $\sup _{[0, T]}\left\{u_{j}(t)-\left.u_{\ell}(t)\right|_{1} \rightarrow 0\right.$ as $j, \ell \rightarrow \infty$. Let $u \in \mathscr{C}_{T}^{1}$ be the limit of $\left\{u_{j}\right\}$. Since every $u_{j}$ is a weak solution of (1.1) with data $u_{j}^{0}, u_{j}^{1}$ and $f_{j}$, it follows that $u$ is a weak solution of (1.1).

We note that (4.16) implies

$$
|u(t)|_{E} \leqslant|u(0)|_{E}+\int_{0}^{t}\left[\|f(\tau)\|+d_{2}|u(\tau)|_{E}\right] \mathrm{d} \tau
$$

With (4.3) it follows that

$$
|u(t)|_{1} \leqslant d_{6}\left(|u(0)|_{1}+\int_{0}^{t}\left[\|f(\tau)\|+|u(\tau)|_{1}\right] \mathrm{d} \tau\right) \text { for } t \in[0, T] .
$$

This estimate holds for every $u_{j}$ used in the above proof and therefore even for the weak solution $u \in \mathscr{C}_{T}^{1}$ of $(1.1)$.

Consider the solution $u \in H^{2}\left([0, T], L_{2}(\Omega)\right) \cap H^{1}\left([0, T], \stackrel{\circ}{H}^{m}(\Omega)\right)$ of $(4.15)$ constructed above. It holds that

$$
A(t) u(t)=f(t)-u^{\prime \prime}(t)-B(t) u^{\prime}(t)
$$

a.e. in $[0, T]$. We consider a fixed $t \in[0, T]$ and conclude by Lemma 3.1 (applied to functions being constant in $t$ ) that

$$
\|u(t)\|_{2 m} \leqslant d_{7}\left[\|f(t)\|+\left\|u^{\prime \prime}(t)\right\|+\left\|u^{\prime}(t)\right\|_{m}+\|u(t)\|\right] .
$$

Since this inequality holds a.e. in $[0, T]$, we obtain that $u \in L_{2}\left([0, T], H^{2 m}(\Omega)\right)$.

From (4.11), $u_{j}^{\prime}(0) \rightarrow u^{1}, u_{j}^{\prime \prime}(0) \rightarrow u^{2}$ and the construction of the solution $u$ of $(4.15)$ it follows by a standard argument (compare $\S 3.8 .2$ of [10]) that $v:=u^{\prime}$ is a weak solution of

$$
\begin{aligned}
& v^{\prime \prime}(t)+A(t) v(t)+B(t) v^{\prime}(t)=f^{\prime}(t)-A^{\prime}(t) u(t)-B^{\prime}(t) u^{\prime}(t) \text { for } t \in[0, T], \\
& v(t) \in \stackrel{\circ}{H}^{m}(\Omega) \quad \text { for } t \in[0, T], \\
& v(0)=u^{1}, \quad v^{\prime}(0)=u^{2} .
\end{aligned}
$$

Since $f^{\prime}-A^{\prime} u-B^{\prime} u^{\prime} \in L_{2}\left([0, T], L_{2}(\Omega)\right)$, Lemma 4.2 yields the existence of a weak solution $v \in \mathscr{C}_{T}^{1}$ of (4.27). From the uniqueness of $v$ we obtain $u^{\prime}=v$ and hence $u^{\prime} \in \mathscr{C}_{T}^{1}$. With $u \in \mathscr{C}_{T}^{1},(4.25)$ and Lemma 3.1 we conclude that $u \in \mathscr{C}_{T}^{2}$. We apply (4.24) to (4.27) and obtain

$$
\left|u^{\prime}(t)\right|_{1} \leqslant d_{6}\left(\left|u^{\prime}(0)\right|_{1}+\int_{0}^{t}\left\|f^{\prime}(t)-A^{\prime}(t) u(t)-B^{\prime}(t) u^{\prime}(t)\right\| \mathrm{d} t\right) .
$$


With (4.24) and (4.26) it follows that

$$
|u(t)|_{2} \leqslant d_{8}\left(|u(0)|_{2}+\int_{0}^{t}\left[\left\|f^{\prime}(\tau)\right\|+\|f(\tau)\|+|u(\tau)|_{2}\right] \mathrm{d} \tau+\|f(t)\|\right)
$$

for $t \in[0, T]$. Thus we have proved the following lemma.

Lemma 4.3. Let Assumption 1.1 be satisfied for $k=k_{0}$. If $u^{0} \in H^{2 m}(\Omega) \cap \dot{H}^{m}(\Omega)$, $u^{1} \in \stackrel{H}{H}^{m}(\Omega)$ and $f \in H^{1}\left([0, T], L_{2}(\Omega)\right)$, then $(1.1)$ has a unique solution $u \in \mathscr{C}_{T}^{2}$. Furthermore (4.29) holds, where $d_{8}>0$ depends only on $c_{1}, c_{2}, c_{3}$ of Assumption 1.1 and on (1.11), (1.13).

\section{Higher regularity}

We prove Theorem 1.1 by induction with respect to $j$. Instead of $(1.10)$ we prove

$$
\begin{aligned}
|u(t)|_{j} \leqslant & D_{3}\left(|u(0)|_{j}+\int_{0}^{t}\left[\left\|\partial_{i}^{j-1} f(\tau)\right\|+|f(\tau)|_{j-2}+C_{3}|u(\tau)|_{j}\right] \mathrm{d} \tau\right) \\
& +D_{4}|f(t)|_{j-2} \text { for } t \in[0, T],
\end{aligned}
$$

where $C_{3}>0$ depends only on $j, c_{1}, c_{2}$ and $c_{3}$ (of Assumption 1.1) and on (1.11), (1.12), while $D_{3}, D_{4}>0$ depend only on $j, c_{1}, c_{2}, c_{3}$ and on (1.11), (1.13). Note that it follows from (5.1) by Gronwall's lemma that

$$
\begin{aligned}
|u(t)|_{j} \leqslant & \mathrm{e}^{D_{3} C_{3} t}\left(2 D_{3}|u(0)|_{j}+D_{3}\left(2+C_{3} D_{4}\right) \int_{0}^{t}\left[\left\|\partial_{t}^{j-1} f(\tau)\right\|+|f(\tau)|_{j-2}\right] \mathrm{d} \tau\right) \\
& +D_{4}|f(t)|_{j-2} \text { for } t \in[0, T] .
\end{aligned}
$$

Hence (5.1) implies (1.10).

For $j=2$, Theorem 1.1 is proved by Lemma 4.3. In order to prove Theorem 1.1 for $\mathrm{j} \geqslant 3$, we differentiate (1.1) with respect to $t$ (formally) and set $v:=u^{\prime}$. This yields

$$
\begin{aligned}
& v^{\prime \prime}(t)+A(t) v(t)+B(t) v^{\prime}(t)=f^{\prime}(t)-A^{\prime}(t) u(t)-B^{\prime}(t) u^{\prime}(t) \text { for } t \in[0, T] \\
& v(t) \in \dot{H}^{m}(\Omega) \quad \text { for } t \in[0, T] \\
& v(0)=u^{1}, \quad v^{\prime}(0)=u^{2} \\
& u(t)=u^{0}+\int_{0}^{t} v(\tau) \mathrm{d} \tau \quad \text { for } t \in[0, T]
\end{aligned}
$$

with $u^{2}=f(0)-A(0) u^{0}-B(0) u^{1}$. On the other hand, if $(u, v) \in \tilde{\mathscr{C}}_{T}^{j+1} \times \mathscr{C}_{T}^{j}$ is a solution of (5.3), (5.4) (with $j \geqslant 2$ ), then $u \in \mathscr{C}_{T}^{j+1}$ and $u$ solves (1.1). In fact, it follows from $u^{0} \in \dot{H}^{m}(\Omega), v(t) \in \dot{H}^{m}(\Omega)$ for $t \in[0, T]$ and $(5.4)$ that $u(t) \in \dot{H}^{m}(\Omega)$ for $t \in[0, T]$. Furthermore (5.4) implies $u^{\prime}=v$ and $u(0)=u^{0}, u^{\prime}(0)=u^{1}$. Integrating (5.3) with respect to $t$ we obtain that (1.1) holds. Finally, it follows from

$$
A(t) u(t)=f(t)-u^{\prime \prime}(t)-B(t) u^{\prime}(t) \quad \text { for } t \in[0, T]
$$

by Lemma 3.1 that $u \in C\left([0, T], H^{(j+1) m}(\Omega)\right)$. This and $u \in \tilde{\mathscr{C}}_{T}^{j+1}$ imply $u \in \mathscr{C}_{T}^{j+1}$.

Let Theorem 1.1 be proved for $j=2, \ldots, J \leqslant k-1$. We prove the existence of a solution $(u, v) \in \tilde{\mathscr{C}}_{T}^{J+1} \times \mathscr{C}_{T}^{J}$ of (5.3), (5.4) by the method of successive approximations 
(as in [5]). Let $u_{0} \in \tilde{\mathscr{C}}_{T}^{J+1}$ such that $\partial_{t}^{v} u_{0}(0)=u^{v}$ for $v=0, \ldots, J$ (compare Lemma 8.8 of [9]); here $u^{v}$ is defined by (2.7) for $v \geqslant 2$. We define $u_{\mu}$ for $\mu \geqslant 1$ by

$$
\begin{aligned}
& v_{\mu}^{\prime \prime}(t)+A(t) v_{\mu}(t)+B(t) v_{\mu}^{\prime}(t)=f^{\prime}(t)-A^{\prime}(t) u_{\mu-1}(t)-B^{\prime}(t) u_{\mu-1}^{\prime}(t) \text { for } t \in[0, T] \\
& v_{\mu}(t) \in \check{H}^{m}(\Omega) \quad \text { for } t \in[0, T], \\
& v_{\mu}(0)=u^{1}, \quad v_{\mu}^{\prime}(0)=u^{2}, \\
& u_{\mu}(t):=u^{0}+\int_{0}^{t} v_{\mu}(\tau) \mathrm{d} \tau \quad \text { for } t \in[0, T]
\end{aligned}
$$

Then $u_{\mu} \in \tilde{\mathscr{C}}_{r}^{j+1}$ for $\mu=1,2, \ldots$, as can be seen in the following way: assume that $u_{\mu-1} \in \tilde{\mathscr{C}}_{T}^{J+1}$ and $\partial_{\imath}^{v} u_{\mu-1}(0)=u^{v}$ for $v=0, \ldots, J$. Then $\left(u^{1}, u^{2}, f^{\prime}-A^{\prime} u_{\mu-1}-B^{\prime} u_{\mu-1}^{\prime}\right)$ satisfies the compatibility condition for (5.6) of order $J$. Corollary 8.3 of [9] yields

$$
f^{\prime}-A^{\prime} u_{\mu-1}-B^{\prime} u_{\mu-1}^{\prime} \in \tilde{\mathscr{C}}_{T}^{J-1} \subset \mathscr{C}_{T}^{J-2} \cap C^{J-1}\left([0, T], L_{2}(\Omega)\right) \text {. }
$$

By the induction hypothesis we obtain the existence of a unique solution $v_{\mu} \in \mathscr{C}_{T}^{J}$ of (5.6), and (5.7) yields $u_{\mu} \in \tilde{\mathscr{C}}_{T}^{J+1}$. Finally it follows from (5.6) and (5.7) that $\partial_{\tau}^{v} u_{\mu}(0)=u^{v}$ for $v=0, \ldots, J$.

Consider $w_{\mu}:=u_{\mu+1}-u_{\mu}, \tilde{w}_{\mu}:=v_{\mu+1}-v_{\mu}$. It holds

$$
\begin{aligned}
& \tilde{w}_{\mu}^{\prime \prime}(t)+A(t) \tilde{w}_{\mu}(t)+B(t) \tilde{w}_{\mu}^{\prime}(t)=-A^{\prime}(t) w_{\mu-1}(t)-B^{\prime}(t) w_{\mu-1}^{\prime}(t) \\
& \quad \text { for } t \in[0, T], \\
& \tilde{w}_{\mu}(t) \in \stackrel{H}{H}^{m}(\Omega) \quad \text { for } t \in[0, T], \\
& \partial_{t}^{\nu} \tilde{w}_{\mu}(0)=0 \quad \text { for } v=0,1, \ldots, J, \\
& w_{\mu}(t)=\int_{0}^{t} \tilde{w}_{\mu}(\tau) \mathrm{d} \tau \quad \text { for } t \in[0, T]
\end{aligned}
$$

for $\mu \geqslant 1$. By Corollary 8.3 in [9] we obtain

$$
\begin{aligned}
& \left.\left|A^{\prime}(t) w_{\mu-1}(t)+B^{\prime}(t) w_{\mu-1}^{\prime}(t) \tilde{J}_{J-1} \leqslant d_{1}^{\prime}\right| w_{\mu-1}(t)\right|_{J+1} ^{\tilde{1}}, \\
& \left|A^{\prime}(t) w_{\mu-1}(t)+B^{\prime}(t) w_{\mu-1}^{\prime}(t)\right|_{J-2} \leqslant d_{2}^{\prime}\left|w_{\mu-1}(t)\right|_{J}
\end{aligned}
$$

for $t \in[0, T]$. Here and in the following we denote by $d_{1}^{\prime}, d_{2}^{\prime}, \ldots$, positive constants that may depend on $c_{1}, c_{2}, c_{3}$ (of Assumption 1.1), $J$ and on (1.11),(1.12). We apply the induction hypothesis and (5.2) to (5.9). This yields

$$
\begin{aligned}
\left|\tilde{w}_{\mu}(t)\right|_{J} & \leqslant\left. d_{3}^{\prime} \mathrm{e}^{d_{4}^{\prime} t} \int_{0}^{t}\left|w_{\mu-1}(\tau) \tilde{\mid}_{J+1} \mathrm{~d} \tau+d_{5}^{\prime}\right| w_{\mu-1}(t)\right|_{J} \\
& \leqslant\left(d_{3}^{\prime}+d_{5}^{\prime}\right) \mathrm{e}^{d_{4}^{\prime} T} \int_{0}^{t}\left|w_{\mu-1}(\tau)\right|_{J+1} \mathrm{~d} \tau,
\end{aligned}
$$

since $\partial_{t}^{v} w_{\mu}(0)=0$ for $v=0, \ldots, J$ and therefore

$$
\left|w_{\mu-1}(t)\right|_{J} \leqslant \int_{0}^{t}\left|w_{\mu-1}^{\prime}(\tau)\right|_{J} \mathrm{~d} \tau \leqslant \int_{0}^{t}\left|w_{\mu-1}(\tau)\right|_{J+1} \mathrm{~d} \tau
$$

for $t \in[0, T]$. 
From (5.10) and (5.13) we conclude that

$$
\begin{aligned}
\mid w_{\mu}(t) \tilde{\mid}_{J+1} & =\left\|w_{\mu}(t)\right\|_{J m}+\left|w_{\mu}^{\prime}(t)\right|_{J} \\
& \leqslant \int_{0}^{t}\left\|\tilde{w}_{\mu}(\tau)\right\|_{J m} \mathrm{~d} \tau+\left|\tilde{w}_{\mu}(t)\right|_{J} \\
& \leqslant\left(d_{3}^{\prime}+d_{S}^{\prime}\right)(1+T) \mathrm{e}^{d_{4} T} \int_{0}^{t} \mid w_{\mu-1}(\tau) \tilde{J}_{J+1} \mathrm{~d} \tau .
\end{aligned}
$$

Let $K(T):=\left(d_{3}^{\prime}+d_{5}^{\prime}\right)(1+T) \mathrm{e}^{d_{4} T}$. By induction it follows that

$$
\left|w_{\mu}(t) \tilde{\mid}_{J+1} \leqslant K(T)^{\mu} \frac{t^{\mu-1}}{(\mu-1) !} \int_{0}^{T}\right| w_{0}(\tau) \tilde{J_{J+1}} \mathrm{~d} \tau \quad \text { for } t \in[0, T] .
$$

Note that $w_{\mu}=u_{\mu+1}-u_{\mu}, w_{\mu}^{\prime}=v_{\mu+1}-v_{\mu}$. Hence we obtain from (5.16) that

$$
\begin{aligned}
\left|v_{\mu}(t)-v_{v}(t)\right|_{J} & \leqslant \mid u_{\mu}(t)-u_{v}(t) \tilde{\mid}_{J+1} \\
& \leqslant C \sum_{l=v}^{\mu-1} K(T)^{l} \frac{T^{l-1}}{(l-1) !} \text { for } \mu>v, t \in[0, T]
\end{aligned}
$$

with some $C>0$. Hence $\left\{\left(u_{\mu}, v_{\mu}\right)\right\}$ converges in $\tilde{\mathscr{C}}_{T}^{J+1} \times \mathscr{C}_{T}^{J}$. From (5.6), (5.7) it follows that the limit $(u, v) \in \tilde{\mathscr{C}}_{T}^{J+1} \times \mathscr{C}_{T}^{J}$ is a solution of (5.3), (5.4). Thus $u \in \mathscr{C}_{T}^{J+1}$ and $u$ is a solution of (1.1) by the considerations following (5.4).

It remains to prove (5.1) for $j=J+1$. In order to apply (5.1) with $j=J$ to (5.3) we note that

$$
\begin{aligned}
&\left\|\partial_{t}^{J-1}\left[A^{\prime}(t) u(t)+B^{\prime}(t) u(t)\right]\right\|+\left|A^{\prime}(t) u(t)+B^{\prime}(t) u(t)\right|_{J-2} \\
& \leqslant\left|A^{\prime}(t) u(t)+B^{\prime}(t) u(t)\right|_{J-1} \leqslant d_{6}^{\prime}|u(t)|_{J+1}, \\
&\left|A^{\prime}(t) u(t)+B^{\prime}(t) u(t)\right|_{J-2} \leqslant d_{1}|u(t)|_{J} \\
& \leqslant d_{1}\left(|u(0)|_{J}+\int_{0}^{t} \mid u(\tau) \tilde{J_{J+1}} \mathrm{~d} \tau\right)
\end{aligned}
$$

by Corollary 8.3 of [9]; here $d_{1}$ depends on $J, c_{1}, c_{2}, c_{3}$ and on (1.11), (1.13). Note that $v=u^{\prime}$ in (5.3), (5.4). Applying (5.1) with $j=J$ to (5.3) and using (5.18), (5.19), we conclude that

$$
\begin{aligned}
\left|u^{\prime}(t)\right|_{J} \leqslant & \left(D_{3}+D_{4} d_{1}\right)|u(0)|_{J}+D_{3} \int_{0}^{t}\left[\left\|\partial_{t}^{J} f(\tau)\right\|+\left|f^{\prime}(\tau)\right|_{J-2}\right] \mathrm{d} \tau \\
& +\left[D_{3}\left(C_{3}+d_{6}^{\prime}\right)+D_{4} d_{1}\right] \int_{0}^{t}|u(\tau)|_{J+1} \mathrm{~d} \tau+D_{4}\left|f^{\prime}(t)\right|_{J-2} \\
& \text { for } t \in[0, T] .
\end{aligned}
$$

From (5.5) we obtain by Lemma 8.2 of [9] and by Lemma 3.1 that

$$
\|u(t)\|_{(J+1) m} \leqslant d_{2}\left[\|f(t)\|_{(J-1) m}+\left|u^{\prime}(t)\right|_{J-1}+\|u(t)\|\right]
$$

for $t \in[0, T]$, where $d_{2}>0$ depends on $J, c_{1}, c_{2}, c_{3}$ and (1.11), (1.13). Combining (5.1) (with $j=J$ ), (5.20) and (5.21) we conclude that (5.1) holds with $j=J+1$. 


\section{Acknowledgements}

The author is grateful to Professor $\mathbf{R}$. Leis for encouraging this research and to $H$. Koch for intensive discussions.

\section{References}

1. Agmon, S., Lectures on Elliptic Boundary Value Problems, Van Nostrand, New York, 1965.

2. Chen, C. and von Wahl, W., 'Das Rand-Anfangswertproblem für quasilineare Wellengleichungen in Sobolevräumen niedriger Ordnung, J. Reine Angew. Math., 337, 77-112 (1982).

3. Dafermos, C. M. and Hrusa, W. J., 'Energy methods for quasilinear hyperbolic initial-boundary value problems. Applications to elastodynamics', Arch. Rat. Mech. Anal., 87, 267-292 (1985).

4. Faedo, S., 'Un nuovo metodo per l'analisi esistenziale e quantitativa dei problemi di propagazione', Ann. Sc. Norm. Sup. Pisa, 1, (3), 1-40 (1949).

5. Ikawa, M., 'Mixed problems for hyperbolic equations of second order', J. Math. Soc. Japan, 20, 580-608 (1968).

6. Kamke, E., Differentialgleichungen, Lösungsmethoden und Lösungen, Bd. 1, Akademische Verlagsgesellschaft, Leipzig, 1959.

7. Kato, T., Abstract Differential Equations and Nonlinear Mixed Problems, Academia Nazionale Dei Lincei Scuola Normale Superiore di Pisa, Lezione Fermiane, Pisa, 1985.

8. Koch, H., 'Mixed problems for fully nonlinear hyperbolic equations', Math. $Z$., to be published.

9. Lesky, P., 'Local existence for solutions of fully nonlinear wave equations', Math. Meth. in the Appl. Sci., 14, $483-508$ (1991).

10. Lions, J. L. and Magenes, E., Non-Homogeneous Boundary Value Problems and Applications, Springer, Berlin, 1972.

11. Milani, A. J., 'A regularity result for strongly elliptic systems', Boll. U.M.I. 2-B, (6), 641-651 (1983).

12. Strauss, W. A., 'On continuity of functions with values in various Banach spaces', Pacific J. Math., 19, 543-551 (1966). 